\title{
ROGUE COMMUNITY COLLEGE: DISCIPLINE- SPECIFIC ONLINE WRITING LAB WITH 24/7 ACCESS AND ASYNCHRONOUS PEER TUTORING
}

Rogue OWL is a unique, multi-discipline, online writing assistance resource, designed to equalize access for all students, across all digital boundaries. Its design and content is flexible and transferable, and it provides a cost-effective vehicle for addressing a variety of needs at multiple institutions.

The Rogue OWL is a 24/7 online interactive writing support service, which provides writers' resources; research, writing, and document design guides across the curriculum; citation and documentation information; as well as asynchronous peer tutoring. The web-based nature of this program effectively removes time and place barriers for students, staff, and faculty-whether across the hall, or across the country.

In addition to support for individual students, the OWL also equalizes writing assistance access for a multi-campus institution, without duplicating physical services at each satellite campus. The OWL guarantees access to resources to previously underserved populations, including those from economically disadvantaged regions. In developing the final site, special consideration was given to technical capabilities of student users, and the OWL functions equally well, whether a user has sophisticated, updated technology, or relies on lower-end technology. (Note: many student users cannot afford new computers in addition to tuition, books, and fees, so we felt it was vital to compensate for low-end technology in our design strategy.)

The OWL guarantees access to resources to traditional, multi-tasking students, as well as to nontraditional, part-time, and evening students, who are often unable to spend long periods of time on campus to receive assistance in physical writing labs. OWL also directly supports distance learners, who, although able to access online courses, have been previously denied equal access support services, specifically, writing assistance. Furthermore, the OWL directly responds to the writing needs of professional/technical students, with its extensive offerings of level-appropriate modules, tailored to individual discipline requirements, in a broad spectrum of fields, including: Electronic Engineering, Automotive Technology, Emergency Medical Technician, Manufacturing and Welding Technology, and Human Services. The OWL is the first writing lab in the nation to address professional technical fieldspecific writing expectations at the undergraduate level.

The content is standardized and has been evaluated by academicians and professionals in the field, thus it is not tailored to a specific institution's curriculum. It is a highly transferable body of information applicable to all undergraduate institutions, including community colleges, technical and vocational institutes, and universities. Licensing institutions can customize content, including adding, modifying, and deleting - to create a more institution-specific site. The OWL is cost-effective, scalable and transferable, requiring minimal technical administration and no technical infrastructure other than Internet connection. Visit http://www.rogueowl.org. 